\title{
Corrigendum to the paper "The number of solutions of the Mordell equation" (Acta Arith. 88 (1999), 173-179)
}

by

Dimitrios Poulakis (Thessaloniki)

In Lemma 2 we produce an algebraic integer $\xi$ which satisfies some conditions. For our purpose $\xi$ must not be a rational integer. As Professors T. Wooley and M. Bennett pointed out to me this is not obvious by our arguments. So there is a gap in the proof. In this note we give a short proof of Lemma 2 by another method, which yields a significantly better estimate, and we considerably improve the estimates of our Theorems 1 and 2. For any positive integer $a$ we write $\log ^{*} a$ for $\max \{1, \log a\}$ and $\omega(a)$ for the number of its prime divisors.

Lemma 2. Let $D$ be a rational integer with $|D|>1$. Denote by $P(D)$ the product of distinct prime divisors $p$ of $D$ with $p>3$. If $D$ has no prime divisors $>3$ put $P(D)=1$. Then the number of cubic fields (up to isomorphism) of discriminant $D$ is at most $225 P(D)^{1 / 2} \log ^{*} P(D)$.

Proof. If $D$ is a perfect square, then [1, Chapter 6, p. 333] implies that the number of cubic fields (up to isomorphism) of discriminant $D$ is $\leq 2^{\omega(D)-1}$. Suppose now that $D$ is not a perfect square. Then $D=a\left(3^{m} b\right)^{2}$, where $a, b \in \mathbb{Z}, b$ is not divisible by $3, a$ is square free and $m$ a nonnegative integer. It follows from [4, Théorème 2.5] that the number of cubic fields (up to isomorphism) of discriminant $D$ is $\leq 2^{\omega(b)-1} 9 h$, where $h$ is the class number of the quadratic field $\mathbb{Q}(\sqrt{-3 a})$. By $[2$, pp. 620-625] we can take $|D| \geq 23$. Furthermore, [3] implies that $h<5 d^{1 / 2} \log ^{*} d$, where $d$ is the discriminant of $\mathbb{Q}(\sqrt{-3 a})$. Combining the above estimates yields the lemma.

Using the above version of Lemma 2, we obtain the following improved version for Theorem 2 .

Theorem 2. Let $S$ be a finite set of rational primes with $2,3 \in S$.

2000 Mathematics Subject Classification: 11D25, 11G05. 
Denote by $P(S)$ the product of primes $p$ in $S$ with $p>3$. If $S=\{2,3\}$, put $P(S)=1$. Then the number of $\mathbb{Q}$-isomorphism classes of elliptic curves over $\mathbb{Q}$, with good reduction outside of $S$, is

$$
<10^{11 \sharp S+23} P(S)^{1 / 2} \log ^{*} P(S) .
$$

As a consequence of Theorem 2, we get the following improved version for Theorem 1.

Theorem 1. Let $k$ be a nonzero rational integer. Denote by $P(k)$ the product of the prime divisors $p$ of $k$ with $p>3$. If $k$ has no prime divisors $>3$, put $P(k)=1$. Then the number of solutions $(x, y) \in \mathbb{Z}^{2}$ of the equation $y^{2}=x^{3}+k$ is

$$
<10^{11 \omega(k)+45} P(k)^{1 / 2} \log ^{*} P(k) .
$$

\section{References}

[1] H. Cohen, A Course in Computational Algebraic Number Theory, Springer, 1993.

[2] H. Hasse, Number Theory, Springer, Berlin, 1980.

[3] A. F. Lavrik, A remark on the Siegel-Brauer theorem concerning the parameters of algebraic number fields, Mat. Zametki 8 (1970), 259-263 (in Russian); English transl.: Math. Notes 8 (1970), 615-617.

[4] J. Martinet et J. J. Payan, Sur les extensions cubiques non-Galoisiennes des rationnels et leur clôture Galoisienne, J. Reine Angew. Math. 228 (1967), 15-37.

Department of Mathematics

Aristotle University of Thessaloniki

54006 Thessaloniki, Greece

E-mail: poulakis@ccf.auth.gr 